\title{
Progress and problems in massive star pulsation theory
}

\author{
Hideyuki Saio ${ }^{1}$, Cyril Georgy ${ }^{2}$ and Georges Meynet $^{3}$ \\ ${ }^{1}$ Astronomical Institute, Graduate School of Science, Tohoku University, \\ Sendai 980-8578, Japan \\ email: saio@astr.tohoku.ac.jp \\ ${ }^{2}$ Astrophysics, Lennard-Jones Laboratories, EPSAM, Keele University, \\ Staffordshire ST5 5BG, UK \\ email: c.georgy@keele.ac.uk \\ ${ }^{3}$ Geneva Observatory, University of Geneva, Maillettes 51, CH-1290 Versoix, Switzerland \\ email: Georges. Meynet@unige.ch
}

\begin{abstract}
Massive stars pulsate in various modes; radial and nonradial p-modes, g-modes, and strange modes including oscillatory convective (non-adiabatic $\mathrm{g}^{-}$) modes. Those modes are responsible for the light and velocity variations of $\beta$ Cephei stars, slowly pulsating B (SPB) stars, and $\alpha$ Cyg variables. The instability mechanisms for these pulsations are discussed. We also discuss the relation between the evolution of massive stars and the excitation of strange modes, which are considered responsible for the pulsation in most of the $\alpha$ Cyg variables. The surface He and CNO abundances of hotter $\alpha$ Cyg variables seem to indicate that the Ledoux criterion of convection is better than the Schwarzschild criterion, although the latter is extensively used in stellar evolution computations.
\end{abstract}

Keywords. massive stars, pulsation, instability, mixing

\section{Introduction}

There are various types of pulsational variables among massive stars; those are $\beta$ Cephei variables, classical Cepheids, (main-sequence and supergiant) SPB (slowly pulsating B) stars, and $\alpha$ Cyg variables. Figure 1 shows some of those variables in the HR diagram with corresponding theoretical instability boundaries. $\beta$ Cephei stars and classical Cepheids are radial and nonradial p-mode pulsators excited by the $\kappa$-mechanism of the Fe-opacity peak at $\sim 2 \times 10^{5} \mathrm{~K}$ and of the He II opacity peak at $\sim 3 \times 10^{4} \mathrm{~K}$, respectively. SPB stars pulsate in g-modes excited by the same Fe-opacity peak. Strange modes are thought to be responsible for most of the $\alpha$ Cyg variables.

\section{Excitation mechanisms of stellar pulsations}

Stellar pulsations are excited mechanically or thermally. Examples of the mechanical excitation are stochastic excitation by convection in solar-like and red-giant stars, and the excitation by the tidal force in eccentric close binary stars. The excitation mechanisms involving flows of thermal energy (non-adiabatic effects) are $\kappa$ mechanism, oscillatory convective (non-adiabatic $g^{-}$) modes, and strange modes. In this paper, we discuss excitation of pulsations due to non-adiabatic effects, which are thought to be responsible for most of the pulsations in massive stars. 


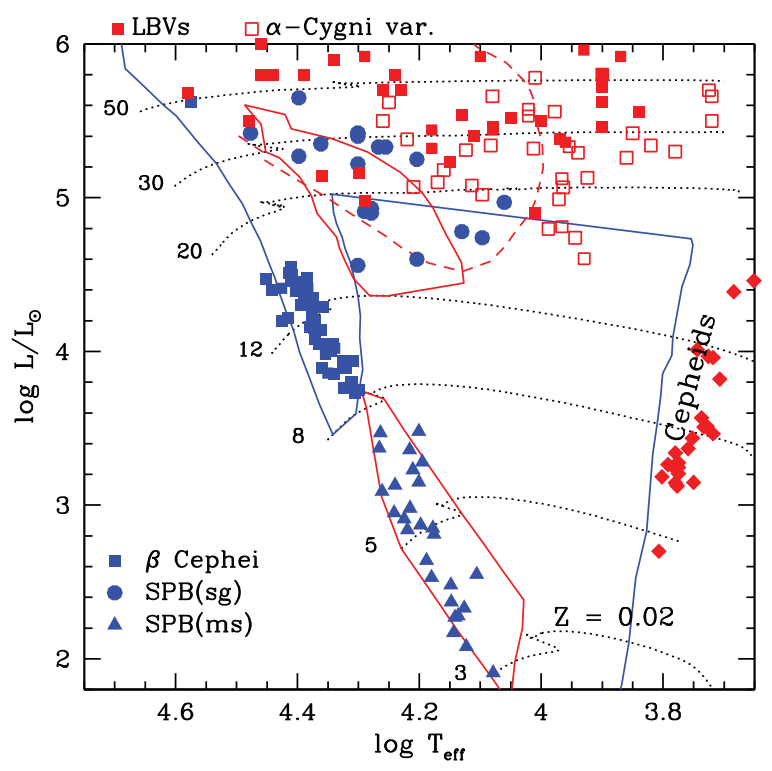

Figure 1. Variable massive stars on the HR diagram and theoretical stability boundaries. Parameters of the classical Cepheids are taken from Turner \& Burke (2002), $\beta$ Cep stars from Sterken \& Jerzykiewicz (1993), Handler et al. (2003), SPB stars from De Cat \& Aerts (2002), Niemczura (2002), Lefever, Puls, \& Aerts (2007), LBVs from van Genderen (2001), $\alpha$ Cyg variables from van Leeuwen, van Genderen, \& Zegelaar (1998).

\section{1. $\kappa$-mechanism}

Driving of stellar pulsation by the $\kappa$-mechanism occurs in layers where matter gains thermal energy in the compressed (hotter) phase and loses energy in the expanded (cooler) phase. The energy gained in the compressed phase makes the following expansion stronger than the previous one, while losing energy in the expanded phase makes the following contraction stronger. Thus, the region helps for the amplitude of pulsation to gradually increase (in other words, thermal energy is gradually converted to pulsation energy); we call the layers driving zone. On the other hand, if the energy gain and loss occurs in the opposite phases, the layers tend to damp the pulsation; we call the layers (radiative) damping zone. If the effect of the driving zone(s) exceeds the effect of damping zone(s) for a pulsation mode in a star, the pulsation will grow to a finite amplitude. Detailed discussion of the $\kappa$-mechanism is given in e.g. Cox (1974).

The energy gain and loss of matter corresponds to blocking and releasing radiation energy from the stellar interior by the effect of opacity $(\kappa)$ variations. For weakly nonadiabatic pulsations, driving occurs in the layers satisfying the inequality

$$
\frac{d}{d r}\left(\kappa_{T}+\frac{\kappa_{\rho}}{\Gamma_{3}-1}\right)>0
$$

(Unno et al. 1989, ch. 5), where $\kappa_{T}=(\partial \ln \kappa / \partial \ln T)_{\rho}, \kappa_{\rho}=(\partial \ln \kappa / \partial \ln \rho)_{T}$, and $\Gamma_{3}-1=$ $(\partial \ln T / \partial \ln \rho)_{S}$ with $S$ being the specific entropy. Roughly speaking, a driving zone satisfying above inequality is located around an opacity peak (as a function of temperature).

The driving or damping is most effective if the thermal time $\tau_{\text {th }}$ defined as

$$
\tau_{\mathrm{th}} \approx \int_{M_{r}}^{M} \frac{C_{\mathrm{v}} T}{L_{\mathrm{rad}}} d M_{r}
$$

is comparable to the pulsation period $\Pi$. Layers with $\tau_{\text {th }} \ll \Pi$ can hardly block radiation 
flow, while layers with $\tau_{\text {th }} \gg \Pi$ hardly absorb or release thermal energy during a cycle of pulsation (i.e., behave nearly adiabatically). In these extreme cases, the $\kappa$-mechanism driving/damping should be very weak. For this reason, the excitation of pulsation by the $\kappa$ mechanism most likely occurs if the relation $\tau_{\text {th }} \sim \Pi$ is met around an opacity peak where inequality (2.1) is satisfied. Then, the radiative damping in layers above (where $\tau_{\text {th }} \ll \Pi$ ) and in layers below (where $\tau_{\text {th }} \gg \Pi$ ) of the driving zone should be small. Therefore, the instability region in the HR diagram associated with an opacity peak generally extends vertically (i.e, with similar $T_{\text {eff }}$ ). The effective temperature is higher for the opacity peak located at a higher temperature.

Nonradial $g$-modes in the SPB stars are excited by the $\kappa$-mechanism of the Fe opacity peak at $T \sim 2 \times 10^{5} \mathrm{~K}$ as for p-modes in the $\beta$ Cep stars. The $\beta$ Cep instability region is hotter than the g-mode (SPB) instability regions, because the periods of SPB stars are longer than those of $\beta$ Cep stars, and hence the condition $\tau_{\mathrm{th}} \sim \Pi$ (at the opacity peak) should be satisfied in slightly deeper layers; i.e., in slightly cooler models.

The SPB instability region in the HR diagram is divided into two parts (main-sequence and supergiant parts; Fig. 1). This is related with the presence of convection in the stellar interior. In a deep (and hence high-density) radiative zone, the amplitude of a g-mode spatially oscillates rapidly with very short wavelengths, which enhances the radiative damping (see e.g. Unno et al.1989 for details). For this reason, g-modes are generally not excited in a star with a radiative core. This is the reason why the distribution of the main-sequence SPB stars is bounded exactly at the end of main-sequence (TAMS) where the convective core disappears. In massive stars of $\gtrsim 12 M_{\odot}$, however, g-modes can be excited even after TAMS because of the appearance of a shell convective zone above the hydrogen burning shell. The shell convection zone can reflect some g-modes, which prevents them from penetrating into the dense radiative core. Therefore, the reflected g-modes are free from strong radiative damping in the core, and are excited even in post-main-sequence models in a certain range of effective temperature (Saio et al. 2006, Godart et al. 2009). The mechanism forms the group of supergiant SPBs separated from the main-sequence SPB stars (Fig. 1).

The period ranges of the excited g-modes in some models are shown in Fig. 2 (open triangles for $\ell=1$ and open squares for $\ell=2$ ). For $M=30$ and $16 M_{\odot}$, g-modes are excited in the supergiant region after TAMS, while g-modes are excited only in the main-sequence stage for $M=6$ and $4 M_{\odot}$.

\subsection{Oscillatory convective modes}

In the linear instability analysis, the occurrence of convection corresponds to the presence of monotonically unstable modes, which are called $\mathrm{g}^{-}$modes with purely imaginary eigenfrequencies in the regime of nonradial pulsations. Shibahashi \& Osaki (1981) found that $\mathrm{g}^{-}$modes of $\ell=10$ associated with the He II convection zone become oscillatory (i.e. having a non-zero real part of frequency) due to the very non-adiabatic condition in very luminous models $\left(12 M_{\odot}, \log L / L_{\odot}=5\right)$ around the cepheid instability strip. We call them oscillatory convective modes in this paper.

Thirty years later, it is found that possibly observable global (i.e., $\ell \leqslant 2$ ) oscillatory convective modes are present associated with the convection zone caused by the Feopacity peak in massive stars (Saio 2011). Although kinetic energy of these modes are generally confined into the convection zone, the amplitude at the surface can be comparable to the amplitude in the convection zone. Dashed line in Fig. 1 shows the region where the amplitude of oscillatory convective modes of $\ell=1$ is larger than $20 \%$ of the maximum amplitude in the convective zone and hence they are likely observable. Figure 2 shows periods of oscillatory convective modes (filled symbols) in evolution models 


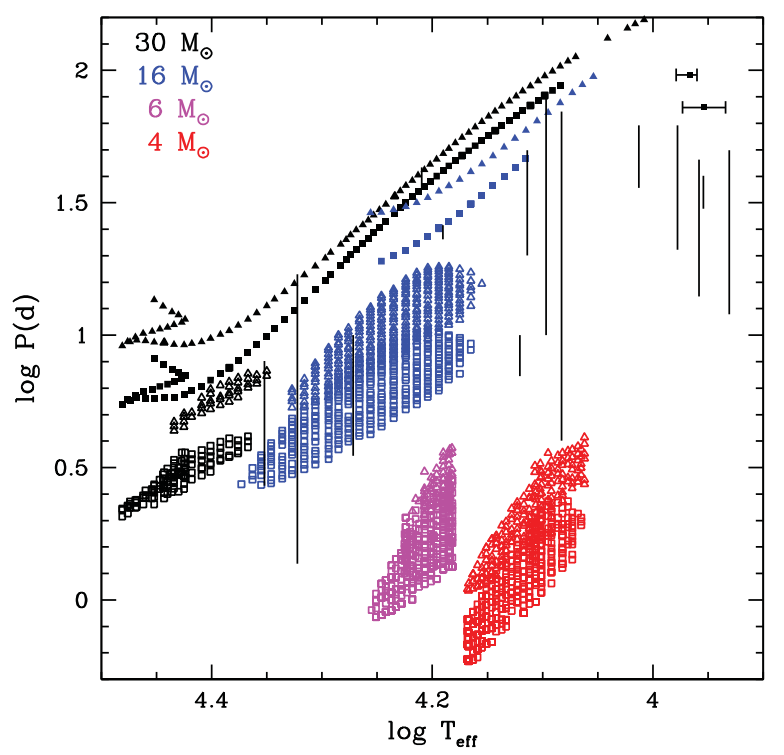

Figure 2. Periods of excited g-modes (open symbols) and oscillatory convective modes (filled symbols) in some models with solar composition evolving from ZAMS to red-giant stage. Triangles are for $\ell=1$, while squares are for $\ell=2$. Oscillatory convective modes whose surface amplitude is larger than $20 \%$ of the maximum amplitude in the convective zone are plotted. Also shown are period ranges of some $\alpha$ Cyg variables. For the 6 and $4 M_{\odot}$ models, nonradial g-modes are excited during the main-sequence evolution with a convective core, while for 16 and $30 M_{\odot}$ models g-modes can be excited even after TAMS because a shell convection zone prevents g-mode amplitude penetrating into the radiative core region. Observed period ranges of $\alpha$ Cyg variables are taken from Richardson, et al. (2011), Kaufer, et al. (1997), Sterken (1977), Moravveji, et al. (2012), van Leeuwen, van Genderen, \& Zegelaar (1998), Sterken, et al. (1999), Burki, Maeder, \& Rufener (1978), Percy et al. (2008), Lefévre, et al. (2009), Percy \& Welch (1983), and Bresolin et al. (2004).

of $M=16$ and $30 M_{\odot}$, the periods are not very sensitive to the stellar mass and longer than those of g-modes. Although the dashed line in Fig. 1 surrounds a large fraction of the $\alpha$ Cyg variable range in the HR diagram, the periods seem too long for most of the $\alpha$ Cyg variables (Fig. 2).

\subsection{Strange modes}

The presence of so called strange modes, whose frequencies vary with a stellar parameter differently from the ordinary modes, were first recognized in the pulsation of luminous helium stars (Wood 1976, Cox et al. 1980). Those were strongly damped modes and discussed in relation with thermal waves (e.g., Saio et al. 1984).

The presence of different and more important strange modes not associated with thermal waves was discovered by Gautschy \& Glatzel (1990). These strange modes are present even in the extreme condition of diminishing thermal time, and more importantly they are unstable with a large growth rate (i.e. excited very rapidly). After the discovery by Gautschy \& Glatzel (1990), the strange mode and strange mode instability refers to this type of strange modes. The properties of the strange modes are discussed in detail by e.g. Glatzel (1994), Saio, Baker \& Gautschy (1998) (nonlinear effect is discussed by Glatzel (2009)).

The strange behavior of the strange mode frequency as a function of a stellar parameter is caused by a trapping of the amplitude into a zone where radiation pressure $P_{\text {rad }}$ is much larger than the gas pressure $P_{\text {gas }}$; such a zone is produced around an opacity peak 
in a model having $L / M \gtrsim 10^{4} L_{\odot} / M_{\odot}$. Combining the hydrostatic and radiative diffusion equations we obtain a relation as

$$
\frac{d P_{\text {gas }}}{d P}=1-\frac{\kappa}{1.3 \times 10^{4} \mathrm{~cm}^{2} \mathrm{~g}^{-1}} \frac{L_{\mathrm{rad}} / L_{\odot}}{M_{r} / M_{\odot}},
$$

where Rosseland-mean opacity per unit mass $\kappa$ is given in units of $\mathrm{cm}^{2} \mathrm{~g}^{-1}$. This equation indicates that in the envelope of a star with $L / M \gtrsim 10^{4} L_{\odot} / M_{\odot}$, gas pressure would become less important around an opacity peak. If the pulsation amplitude is confined around the opacity peak, $\kappa$-mechanism driving is enhanced and hence such a mode tend to be excited. This is one of the mechanisms for the excitation of strange modes; i.e. an enhancement of $\kappa$-mechanism.

For a sufficiently high $L / M$, the thermal time (eq. (2.2)) in the envelope can be much shorter than the pulsation period. Then, blocking of radiation by the opacity variation becomes ineffective, and hence the $\kappa$-mechanism would not work as discussed above. Even in such a case of very short thermal timescale, strange modes are excited as discussed below. In the limit of short thermal time, the radiative flux perturbation, $\Delta F_{\text {rad }}$, which can be derived from the radiative diffusion equation, diminishes; i.e.,

$$
\frac{\Delta F_{\mathrm{rad}}}{F_{\mathrm{rad}}} \approx-\frac{\kappa_{T}}{4} \frac{\Delta P_{\mathrm{rad}}}{P_{\mathrm{rad}}}-\kappa_{\rho} \frac{\Delta \rho}{\rho}-\frac{\kappa F_{\mathrm{rad}}}{c} \frac{d \Delta P_{\mathrm{rad}}}{\rho d r} \rightarrow 0
$$

where a plane-parallel approximation is used for simplicity, temperature perturbation $\Delta T / T$ is replaced with $\Delta P_{\text {rad }} /\left(4 P_{\text {rad }}\right)$, and $c$ is the speed of light. If $P_{\text {rad }} \gg P_{\text {gas }}, P \approx$ $P_{\text {rad }}$. Then, from equation (2.4), we obtain

$$
\frac{d(f \Delta P)}{d r} \approx-\frac{\kappa_{\rho} \kappa F_{\mathrm{rad}}}{c} f \Delta \rho,
$$

where a function $f$ is defined as

$$
f \equiv \exp \left[(4 c)^{-1} \int^{r} \kappa \kappa_{T} F_{\text {rad }} P^{-1} d r\right] .
$$

The relation between $\Delta P$ and $\Delta \rho$ given in equation (2.5) contains a spatial differentiation in contrast to the algebraic adiabatic relation. In the adiabatic relation no phase difference occurs between $\Delta P$ and $\Delta \rho$, while we expect a large phase difference from equation (2.5). If the perturbation is approximated by a plane wave $\propto \exp (i k r)$, for example, the phase of $\Delta P$ would differ from the phase of $\Delta \rho$ by $90^{\circ}$, which would yield a dynamical type instability. Thus, the relation between $\Delta P$ and $\Delta \rho$ given in equation (2.5) should cause a very rapid instability in the extremely nonadiabatic condition. We note that $\kappa_{T}$ (which plays a decisive role in the $\kappa$ mechanism) is not important at all here, while $\kappa_{\rho}$ plays a decisive role (eq.2.5).

In summary, strange modes whose kinetic energy is trapped in a zone around an opacity peak are excited in two ways; 1 ) enhanced $\kappa$-mechanism driving due to the trapping, 2) dynamical-type instability in the extremely nonadiabatic condition. As the ratio of the thermal time in the trapped zone to the pulsation period decreases, the excitation mechanism shifts from 1) to 2).

\section{Evolution of massive stars and $\alpha \mathrm{Cyg}$ variables}

During the post-main-sequence evolution toward the red-supergiant stage, a massive star with an initial mass $\lesssim 50 M_{\odot}$ do not enter strange-mode instability range in the HR diagram (dashed line in the right panel of Fig. 3) because the mass-luminosity ratio is 

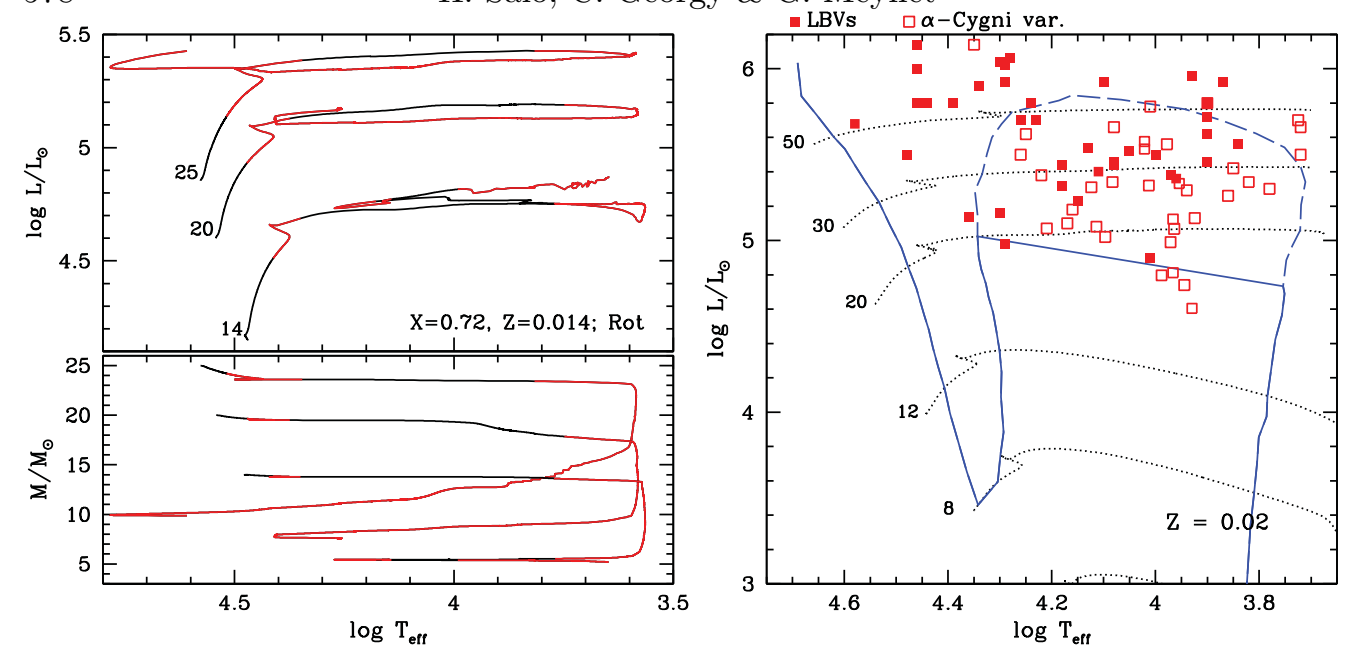

Figure 3. Left panel: evolution tracks in the HR diagram and $\log T_{\text {eff }}-$ mass diagram for 25,20 and $14 M_{\odot}$ models with rotational mixing (adopted from Saio et al. 2013). The parts shown by red lines indicate models in which low-order radial modes are excited. Note that radial pulsations are not excited in the range $4.3 \gtrsim \log T_{\text {eff }} \gtrsim 3.8$ when the stars are evolving toward the red supergiant stage, while they are excited in stars evolving from the red supergiant stage after significant mass is lost. Right panel: Instability boundary of radial pulsations for models evolving toward red supergiant region (dashed line) and for models evolving back to the blue region (solid line) are compared with the distribution of $\alpha$ Cyg variables.

not large enough during the first crossing. However, as the left panel of Fig. 3 indicates, massive stars can come back to the blue supergiant region after losing significant mass in the red supergiant stage. During the second crossing, pulsations are excited due to strange modes in stars whose initial mass is roughly greater than $14 M_{\odot}$, because the ratio $L / M$ has increased due to the substantial mass loss in the red supergiant stage (Saio, Georgy, \& Meynet 2013). Because of the effect of mass loss, the luminosity of the instability boundary for the second crossing decreases by nearly an order of magnitude; i.e., from the dashed line to the solid line in the right panel of Fig. 3. By the shift of the instability boundary, most of the $\alpha$ Cyg variables are included in the instability region. Therefore, we can identify $\alpha$ Cyg variables as stars with the initial mass greater than about $14 M_{\odot}$ evolving in the blue supergiant region after the red supergiant stage where significant mass was lost. (We note that Sonoi \& Shibahashi (2014) showed strange modes still to be excited even if the effect of convection/pulsation interaction is included.)

To justify the evolutionary stage of $\alpha$ Cyg variables, we need observational confirmation for the predicted periods of pulsations and surface compositions modified by the significant mass loss in the red supergiant stage. The left panel of Fig. 3 compare observed period ranges of $\alpha$ Cyg variables with predicted ones for models of $M_{\text {init }}=25 M_{\odot}$ evolving back from the red supergiant stage; blue and red colors are for models based on the Schwarzschild and the Ledoux criterions for the convection boundaries, respectively. As seen in Fig. 3, periods of the excited pulsations roughly cover the observed period ranges of $\alpha$ Cyg variables, irrespective to the convection criterions employed.

However, there are discrepancies between observations and theoretical predictions for the surface CNO and He abundances (right panel of Fig. 3). The surface CNO and He abundances are affected by rotational mixing in the main-sequence stage, dredge up by a deep convective envelope, and mass loss in the red supergiant stage. The evolution models shown in Fig. 3 rotate at $40 \%$ of the critical speed at the ZAMS stage. The surface He abundance and $\mathrm{N} / \mathrm{C}$ and $\mathrm{N} / \mathrm{O}$ ratios increase a little during the main sequence stage 

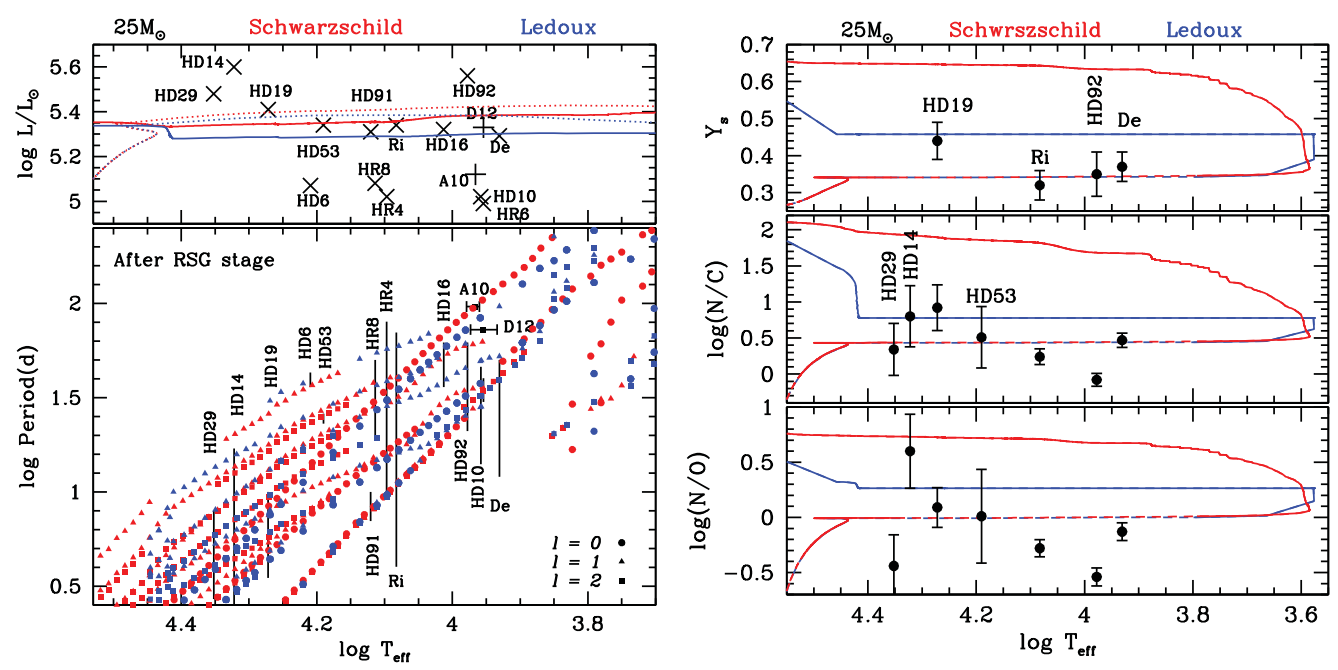

Figure 4. Theoretical periods excited in models $\left(M_{\text {init }}=25 M_{\odot}\right)$ evolved back from the red supergiant stage (left panel), and predicted surface He abundance and N/C, N/O ratios (right panel) are compared with observations. Red and blue lines are for models based on Schwarzschild and Ledoux criterions of convective instability, respectively. In the right panel, solid line parts indicate models in which radial pulsations are excited, while in the dashed line part, no radial pulsations are excited. De $=\operatorname{Deneb}(\alpha$ Cyg $)$, HR6 $=$ HR 685, HD10 $=$ HD 100262, HD92 = HD 92207, HD16 $=$ HD 168607, Ri $=\operatorname{Rigel}(\beta$ Ori $)$, HR $4=$ HR 4338, HR $8=$ HR 8020, HD91 $=$ HD 91619, HD53 = HD 53138, HD6 = HD 662150, HD19 = HD 190603, HD14 = HD 14956, and HD29 = HD 2905 are blue supergiants in the Milky Way. Surface abundances are adopted from Przybilla, et al. (2010), Crowther, Lennon, \& Walborn, and Searle et al. (2008). A10 and D12 are stellar identities used by Bresolin et al. (2004) for stars in NGC 300.

due to the rotational mixing, while they are almost constant after TAMS to the red supergiant stage because of a short evolution time. Up to this stage the difference in the convective criterion causes no effect on the surface compositions.

After the red supergiant stage, where significant mass lost, models evolve back to blue supergiant region with surface He abundance, and N/C, N/O ratios being considerably increased. The degree of the increase depends strongly on the convection criterion adopted; models based on the Schwarzschild criterion have much higher He abundance and $\mathrm{N} / \mathrm{C}, \mathrm{N} / \mathrm{O}$ ratios compared to the models with the Ledoux criterion (see Georgy et al.2014 for more discussion).

For relatively hotter $\alpha$ Cyg variables with $\log T_{\text {eff }}>4.15$, the surface abundances seem roughly consistent with the predictions of models evolved back from the red supergiant stage (probably except for HD 2905) with the Ledoux criterion. (HD 2905 might be just after TAMS and pulsate in non-radial g-modes; Fig. 2.) This indicates, if confirmed, that the Ledoux criterion should be used in stellar evolution calculations although the Schwarzschild criterion are widely used.

However, the surface compositions of relatively cooler $\alpha$ Cyg variables shown in Fig. 3 do not agree with models evolved from the red supergiant stage; the observed He abundances and $\mathrm{N} / \mathrm{C}, \mathrm{N} / \mathrm{O}$ ratios are too small, which are rather consistent with models evolving toward the red supergiant stage but such models are predicted not to pulsate. Very irregular light curves of these stars could be caused by wind variations or g-modes trapped in the envelope as discussed in Gautschy (2009).

Relatively cool blue supergiants A10 and D12 in NGC 300 show clear radial pulsations (Bresolin et al. 2004), which can be explained only by models with reduced masses evolved 
through the red supergiant stage. Dziembowski \& Sławińska (2005) have also found that the masses must be significantly reduced to explain pulsations of A10 and D12. Therefore, the surface He and CNO abundances of the two stars are critically important, because the results would determine which convective criterion, Schwarzschild or Ledoux, should be used in stellar evolution calculations.

\section{References}

Cox, J. P. 1974, Rep. Prog. Phys., 37, 563

Cox, J. P., King, D. S., Cox, A. N., Wheeler, J. C., Hansen, C. J., \& Hodson, S. W. 1980, Space Sci. Rev., 27, 529

Bresolin, F, Pietrzyński, G; Gieren, W. Kudritzki, R.-P., Przybilla, N., \& Fouqu e, P. 2004, ApJ, 600,182

Burki, G., Maeder, A., \& Rufener, F. 1978, A\&\&A, 65, 363

Crowther, P. A., Lennon, D. J., \& Walborn, N. R. 2006, A\& A, 446, 279

De Cat, P. \& Aerts, C. 2002, A\&SA, 393, 965

Dziembowski, W. A. \& Sławińska, J. 2005, AcA, 55, 195

Gautschy, A. 2009, A\&BA, 498, 273

Gatuschy, A. \& Glatzel, W. 1990, MNRAS, 245, 597

Georgy, C., Saio, H., \& Meynet, G. 2014, MNRAS, 439, L6

Glatzel, W. 1994, MNRAS, 271, 66

Glatzel, W. 2009, CoAst, 158, 252

Godart, M., Noels, A., \& Dupret, M.-A., \& Lebreton, Y. 2009, MNRAS, 396, 1833

Handler, G., Shobbrook, R. R., Vuthela, F. F., Balona, L. A., Rodler, F., \& Tshenye, T. 2003, $M N R A S, 341,1005$

Kaufer, A., Stahl, O., Wolf, B., Fullerton, A. W., Th. Gaäg, Th., Gummersbach, C. A., Jankovics, I., Kovács, J., Mandel, H., Peitz, J., Rivinius, Th., \& Szeifert, Th. 1997, A\&A, 320, 273

Lefever, K., Puls, J., \& Aerts, C. 2007, A\& A, 463, 1093

Lefévre, L., Marchenko, S. V., Moffat, A. F. J., \& Acker, A. 2009, A\& A, 507, 1141

Moravveji, E., Guinan, E. F., Shultz, M., Williamson, M. H., \& Moya, A. 2012, ApJ, 747, 108

Niemczura, E. 2002, ASPConf, 259, 230

Percy, J. R. \& Welch, D. 1983, PASP, 95, 491

Percy, J. R., Palaniappan, R., Seneviratne, R., Adelman, S. J., \& Markova, N. 2008, PASP, 120, 311

Przybilla, N., Firnstein, M., Nieva, M. F., Meynet, G., \& Maeder, A. 2010, A\& $\&$, 517, A38

Richardson, N. D., Morrison, N. D., Kryukova, E. E., \& Adelman, S. J. 2011, ApJ, 141, 17

Saio, H. 2011, MNRAS, 412, 1814

Saio, H., Baker, N. H., \& Gautschy, A. 1998, MNRAS, 294, 622

Saio, H., Georgy, C., \& Meynet, G. 2013, MNRAS, 433, 1246

Saio, H., Kuschnig, R., Gautschy, A., Cameron, C., Walker, G. A. H., Matthews, J. M., Guenther, D. B., Moffat, A. F. J., Rucinski, S. M., Sasselov, D., \& Weiss, W. W. 2006, ApJ, 650, 1111

Saio, H., Wheeler, J. C., \& Cox, J. P. 1984, ApJ, 281, 318

Searle, S. C., Prinja, R. K., Massa, D., \& Ryans, R. 2008, A\& A, 481, 777

Shibahashi, H. \& Osaki, Y. 1981, PASJ, 33, 427

Sonoi, T. \& Shibahashi, H. 2014, PASJ, 66, 69

Sterken, C. 1977, A\& $A, 57,361$

Sterken, C., Arentoft, T., Duerbeck, H. W., \& Brogt, E. 1999, A\&\&A, 349, 532

Sterken, C. \& Jerzykiewicz, M. 1993 SSRv, 62, 95

Turner, D. G. \& Burke, J. F. 2002, AJ, 124, 293

Unno, W., Osaki, Y., Ando, H., Saio, H., \& Shibahashi, H. 1989, Nonradial Oscillations of Stars (University of Tokyo Press, Tokyo)

van Genderen, A. M. $2001 A \mathscr{E} A, 366,508$

van Leeuwen, F., van Genderen, A. M., \& Zegelaar, I. 1998, A\& AS, 128, 117

Wood, P. 1976, MNRAS, 174, 531 